\title{
UHPLC-MS/MS Method for the Analysis of 2,6 Toluene Diisocyanate and 2,4 Toluene Diisocyanate Released from Microa-gglomerated Corks in Wine
}

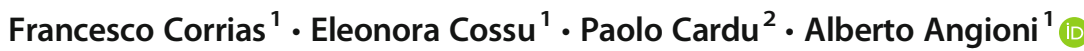 \\ Received: 8 May 2020 / Accepted: 16 September 2020 / Published online: 9 October 2020 \\ (C) The Author(s) 2020
}

\begin{abstract}
Micro-agglomerate corks, made by agglutination of cork granulate through the addition of different adhesives, represent an important slice of the market of cork stoppers. Binder glues which are polyurethane- or butadiene-based have been used since they have strong agglomerating effect. Unfortunately, polyurethane-based glues can have isocyanide end group compounds which can migrate into the wine. 2,4-toluene diisocyanate (2,4-TDI) and 2,6 toluene diisocyanate (2,6-TDI), can be found in adhesive and could migrate into wine. A simple ultrahigh-performance liquid chromatography-mass spectrometry (UHPLC-MS/ MS) method for the determination of these active ingredients (a.is.) in wine has been developed. The method has been validated under Eurachem CITAC guidelines (Cooperation on International Traceability in Analytical Chemistry). Instrument limit of detection (LOD) and to a limit of quantification (LOQ) for 2,6 TDI and 2,4 TDI were 0.42 and 0.39 $\mu \mathrm{g} / \mathrm{L}$, and 1.72 and 1.57 $\mu \mathrm{g} / \mathrm{L}$, respectively. Four different solvents applied for recoveries showed quite different rates ranging for 2,6 TDI and 2,4 TDI from 17.96 to $88.53 \%$, and 40.08 to $99.18 \%$, respectively. Real sample analysis showed low residue levels, especially of 2,6 TDI, with values always below the LOQ. The data reported on real samples allowed to establish that from a risk management purpose, no toxicology risk can be accomplished.
\end{abstract}

Keywords 2,4 TDI $\cdot$ 2,6 TDI $\cdot$ LC-MS/MS $\cdot$ Micro-agglomerated corks $\cdot$ Wine

\section{Introduction}

The use of cork as stoppers is traced back to the ancient Romans; in fact, in the Greek and Roman world, it was customary to use it for the closure of amphorae (Denecker and Vandorpe 2007).

In a bottle of wine, the type of closure represents one of the most important and delicate parts (especially in the case of fine and aging wines). Nowadays, wine bottle can use a different

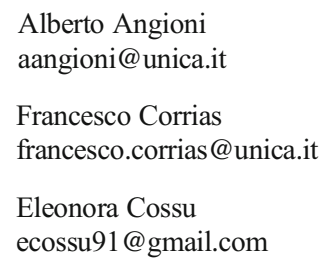

1 Department of Life and Environmental Science, Chemical food Analysis Laboratory, University of Cagliari, University Campus of Monserrato, S.S. 554 - Bivio Monserrato - Sestu. S.P. Monserrato Sestu Km 0, 700 Cagliari, Italy

2 via Dettori 58, 09067, Elmas Cagliari, Italy device for their closing; the most used are cork, screwcaps, and synthetic polymer material such as Low-Density Polyethylene (LDPE); the use of one or the other is related to commercial purpose and wine philosophy (Godden et al. 2008).

Screwcaps have been initially used in the whisky industry with excellent results, and have been introduced in the wine market in the late 1950s in France, after replacing a thin cork layer underneath the cap with neutral polyvinylidene dichloride (PVDC) (Courtney 2001). Their use has been justified from the assertion that they do not allow oxygen to enter the bottle and ensures that the wine remains crisp and wellpreserved; moreover, no corkscrew is required for extracting the closure.

LDPE stoppers, also called synthetic corks, have been improved after their first appearance. Actually, there is a wide variety of synthetic stoppers with different rates of oxygen transmission, promoting a different approach to sensory characteristics of wine (Wirth et al. 2012). A major benefit of synthetic cork is the absence of cork taint (TCA) caused by the different fungi species that lives in natural cork trees (Prak et al. 2007). 
Some authors and experts believe that cork is the best closure for wine, because the small amount of oxygen which permeates through the stopper inside the bottle inhibits the formation of a reduction environment and the development of sulfur compound odors (He et al. 2013). Moreover, corkfinished wines seem to age more gracefully. The tannins become softer and bitterness, especially in white wines, fades with time, and tannins and other substances released from the cork can react with short-chained catechins removing bitterness and harmonize with the compounds naturally present in wine improving mouthfeel (Azevedo et al. 2014; Gil et al. 2006).

On the other hand, in addition to the possible risk of TCA contamination, the production of natural cork is no longer able to support the demand for corks necessary for the wine sector, and therefore, younger and lower-quality corks are used (Pereira et al. 2012), putting the content of the bottle of wine at greater risk. For this reason, some alternatives that seek to exploit processing waste to produce high-efficiency caps have been adopted (Gil 2014).

Cork, authorized for food use, can be natural one-piece cork, natural multi-piece corks made from two or more pieces of natural cork and glued together through an adhesive, natural cork filled in which the lenticels have been filled with cork powder obtained by finishing the natural corks, and technical corks made of a part of an agglomerated cork with on the top and the bottom slice of natural cork assembled using suitable agglutinins.

Moreover, agglomerated caps entirely made of cork granules obtained from byproducts deriving from the processing of natural corks placed together through an adhesive, and microagglomerated caps which have been defined, according to the ISO 633:2019 standard: "cork obtained by agglutination of cork granulate, with a grain size between 0.25 and $8 \mathrm{~mm}$, with adhesives, composed of at least $51 \%$ of granulate of cork (by weight)" (Nasini and Smedile 2012; ISO 633:2019).

Micro-agglomerate corks are made starting from a cork granulate, obtained from the previously boiled plank, in which only the best and lightest part is present, eliminating all the hard or woody parts, and dividing it according to the specific weight (SW) (only granules with a lower SW, between 45-70 $\left.\mathrm{kg} / \mathrm{m}^{3}\right)$ and length $(0.25-8 \mathrm{~mm})$. In order to join and mix the granules, binders such as polyurethane- or butadiene-based glues are still used. These products show strong agglomerating effect and can confer to the caps' resistance to twisting and compression. Unfortunately, polyurethane-based glue often can have isocyanide end groups (Six et al. 2002).

The European Committee for Food Contact Materials and Articles has subscribed a statement concerning the substance which can be found in cork stoppers intended for wine use, reporting a DL of $0.01 \mathrm{mg} / \mathrm{Kg}$ (sum of NCO) for 22,4-TDI (CAS: 584-84-9) and 2,6-TDI (CAS: 91-08-7) (CD-P-MCA 2007). The Environmental Protection Agency (EPA, U.S
2011) established an Action Plan on TDI compounds to assess peoples' exposures, while the Food and Drug Administration (FDA) regarding the toxicological impact of diisocyanate in the cork industry expressed a FCN (finding of no significant impact) (FDA 2017), intending that these compounds will not significantly affect the quality of the human environment.

The International Agency for Research on Cancer (IARC) reported that "there is sufficient evidence for the carcinogenicity of toluene diisocyanate in the experimental animal while there is inadequate evidence in humans," classifying TDI as a $2 B$ carcinogen (IARC 1986).

Several methods for the analysis of 2,4-TDI and 2,6-TDI have been reported in literature to detect the levels in air or in material which can release these compounds in the environment. Most of them uses liquid chromatography UVQDa or MS/MS technique after derivatization (Donchenko et al. 2020; Gagné et al. 2003); others used HPLC-FL (Yeh et al. 2002), or gas chromatography coupled with flame ionization detector (FID) or electron capture detector (ECD) (Yun et al. 2006).

Any paper has been found dealing with the presence of residues of NCO in micro-agglomerate corks or with the possible release of these compounds in wine.

Considering the high use of these stoppers in the modern wine industry, it is compulsory to have a reliable analytical method for the determination of $\mathrm{NCO}$ compounds in cork and to evaluate the amount of possible release in wine.

The aim of this study was to develop and validate a method for the quantification of 2,4-TDI and 2,6-TDI in microagglomerate cork in wine according to Eurachem CITAC Guidelines (Magnusson and Örnemark 2014).

Samples of cork were spiked with appropriate volume of NCO standard solution and extracted with three different solvent solutions and wine; moreover, release tests were performed to study the behavior of 2,4-TDI and 2,6-TDI in wine during a simulated bottle storage.

Finally, the effectiveness of the proposed validated method was evaluated in real micro-agglomerate cork samples. Quantitative analyses have been carried out by LC-MS/MS technique without derivatization step and cleanup.

\section{Materials and Methods}

\section{Chemicals}

Acetone and methanol $(\mathrm{MeOH})$ were LC/MS grade solvents (Sigma-Aldrich, Milan, Italy). Formic acid (> 95\%), acetic acid glacial (99\%), and ethanol (96\%) were reagent grade (Sigma-Aldrich, Milan, Italy), ammonium formate solution $5 \mathrm{M}(0.315 \mathrm{~g} / \mathrm{mL})$ (G1946-85021, Agilent Technologies). MilliQ water with a conductivity less than $18.2 \mathrm{M} \Omega$, was 
prepared using an integrated Milli-pore purification system (MilliQ integral, Merck, Milan, Italy).

Tartaric acid, glycerol, 2,4 toluene diisocyanate, and 2,6 toluene diisocyanate were analytical standards (SigmaAldrich, Milan, Italy).

\section{Preparation of Stock Solutions and Calibration Standards}

Stock solution of the active ingredients (a.is.) diisocyanate standards were prepared by weighting an appropriate aliquot of standard in a 10-mL volumetric flask. 2,4 TDI was prepared at $1412 \mathrm{mg} / \mathrm{L}$ in a solution of MeOH acidified with acetic acid at $5 \%$, while $2,6 \mathrm{TDI}$ at $1207 \mathrm{mg} / \mathrm{L}$ was prepared in acetone. The working solutions were prepared daily by diluting the stock solutions with each matrix extracting solution.

Calibration curves were prepared with five point in eluent mixture, synthetic wine, ethanol, and white wine unpolluted matrix.

\section{Recovery and Release Tests}

Available micro-agglomerate corks were not certified for the absence of glue free from the a.is.; therefore, release and recovery tests have been carried out on one-piece cork, previously spiked with a known amount of 2,4-TDI and 2,6-TDI.

Acid water (tartaric acid at $46 \mathrm{mg} / \mathrm{L}$ until $\mathrm{pH}$ 3.6), synthetic wine (tartaric acid at $46 \mathrm{mg} / \mathrm{L}$, plus $120 \mathrm{~mL}$ of ethanol, $5 \mathrm{~g}$ of glycerol, and $880 \mathrm{~mL}$ of MilliQ water, final $\mathrm{pH} 3.6$, and alcohol content $12 \%$ vol), white wine (Nuragus white wine at $10.5 \%$ vol), and ethanol (96\%) were used as extracting solvents.

Recovery Test Three one-piece corks of $2.5 \mathrm{~cm}$ in diameter and $3.3 \mathrm{~cm}$ in length for each extracting solvent were drilled along the longitudinal axis, to produce four holes equidistant from the central axis with a maximum depth of $1.0 \mathrm{~cm}$, for each cap. Subsequently $20 \mu \mathrm{L}$ of the stock standard solution of 2,4-TDI and 2,6-TDI was added inside the holes; the corks were left to rest in the dark at ambient temperature $\left(18^{\circ} \mathrm{C}\right)$ for $4 \mathrm{~h}$. The corks were then cut in two identical halves for a total of six replicates for each extracting solvent. Each halve was reduced to smaller particles with a stainless-steel cutter, and subsequently ground with a coffee grinder (Clatronic, Cagliari, Italy), being careful that the temperature of the ground cork did not rise. The obtained grinded halves were weighed in $50-\mathrm{mL}$ Falcon plus $30 \mathrm{~mL}$ of extraction solvent, stirred in vortex for $3 \mathrm{~min}$, placed in a rotating carousel for $30 \mathrm{~min}$ and finally centrifuged for $15 \mathrm{~min}$ at $4000 \mathrm{rpm}$ and 10 ${ }^{\circ} \mathrm{C}$ temperature. After centrifugation, the solvent was recovered and transferred in a vial for UHPLC/MS-MS analysis.
Release Test One-piece cork stoppers spiked as previously reported were immersed in the best extracting solvents from the previous recovery test to evaluate the release of the a.is. from whole stoppers. Whole cork placed in a $100-\mathrm{mL}$ screwcapped flask plus $30 \mathrm{~mL}$ of white wine or ethanol for $48 \mathrm{~h}$ under agitation at room temperature and in the dark. Trials were carried out in triplicate.

Reference standards for each test were prepared by adding the same amount of analytical standard placed in the stoppers to $30 \mathrm{~mL}$ of extraction solvent, and to an equal volume of the eluent mixture at $T=0$.

\section{Instrumentation and Analytical Conditions}

An UHPLC 1290 Infinity, coupled with a 6470 Triple Q MSMS Agilent (Agilent, Milan, Italy) system, was used. The column was a Kinetex $\mathrm{C} 18(5 \mu \mathrm{m}, 250 \times 4.6 \mathrm{~mm})$, with a flow of $0.5 \mathrm{~mL} / \mathrm{min}$. Injection volume was of $5 \mu \mathrm{L}$, and samples were analyzed with a binary gradient as follows: solvent A MilliQ at $0.1 \%$ formic acid and $5 \mathrm{mM}$ ammonium formate, solvent B $\mathrm{MeOH}$ at $0.1 \%$ formic acid and $5 \mathrm{mM}$ ammonium formate, $T$ $=0 \min \mathrm{A} 70 \%, T=10 \mathrm{~min}$ A $50 \%, T=15 \mathrm{~min}$ B $100 \%$, total run time $15 \mathrm{~min}$, and postrun $15 \mathrm{~min}$ at initial conditions.

The instrumental limits of detection (LOD) and quantification (LOQ) were calculated as three, and ten times the signal/ noise ratio $(\mathrm{S} / \mathrm{N})$.

Analyses were carried out in MRM mode with the following instrumental conditions: gas temperature $350{ }^{\circ} \mathrm{C}$, gas flow $10 \mathrm{~L} / \mathrm{min}$, nebulizer $30 \mathrm{psi}$, sheath gas temp: $350{ }^{\circ} \mathrm{C}$, sheath gas flow: $12 \mathrm{~L} / \mathrm{min}$, capillary negative $3000 \mathrm{~V}$.

\section{Method Validation}

The analytical method was validated according to Eurachem CITAC Guidelines (Magnusson and Örnemark 2014) assessing linearity, selectivity, precision, method detection limits (LOD), quantitation limits (LOQ), recovery, uncertainty, and matrix effect.

Recovery tests were carried out with six replicates for each solvent at a spiking level of $10 \times$ LOQ. Instrumental repeatability $\left(\mathrm{RSD}_{\mathrm{r}}\right.$, intraday $\left.n=6\right)$, and reproducibility $\left(\mathrm{RSD}_{\mathrm{wR}}\right.$, interday, 6 days, two replicate/day, $n=12$ ) were calculated at two concentrations 2.0 and $7.8 \mu \mathrm{g} / \mathrm{L}$. Each sample belonged from an independent experiment. Instrumental sequence was conducted according to SANTE guidelines indications (SANTE 2016). The matrix effect was evaluated by comparing the analytical responses of the active ingredients in eluent mixture at $T=0$, with those in the extracting solvents. Linearity was assessed analyzing five standard calibration curves performed in triplicate, both in solvents and blank control matrix, and was admitted as acceptable when the coefficient of determination was above 0.990 . Selectivity was evaluated comparing extracts from wine matrices with wine 
Fig. 1 MRM chromatograms of (a) white wine, and (b) white wine spiked at $2 \mu \mathrm{g} / \mathrm{L}$

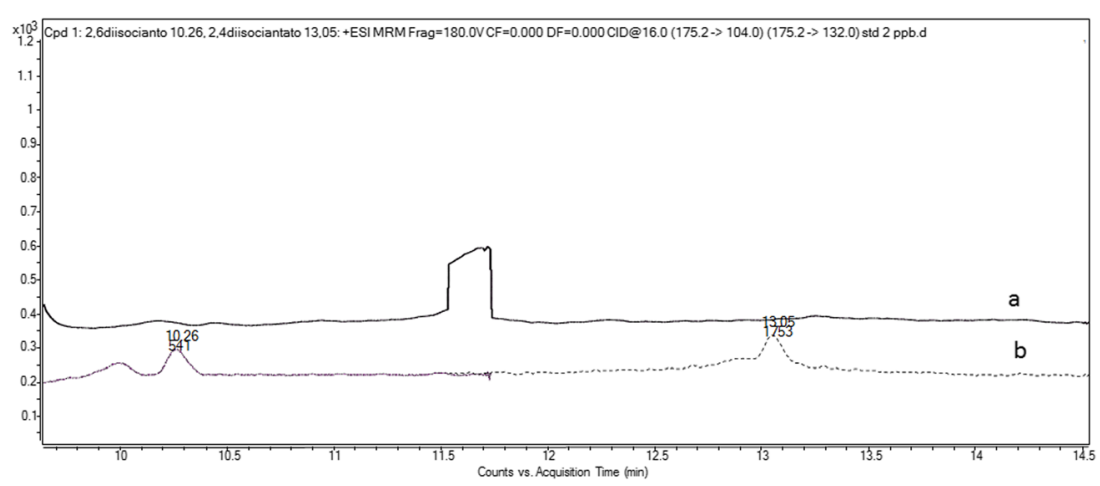

spiked at $2 \mu \mathrm{g} / \mathrm{L}$. Absence of peaks at the retention times of the a.i. was a criterion for confirmation method selectivity. The expanded measurement uncertainty $\left(U^{\prime}\right)$ was calculated by multiplying the combined uncertainty $\left(u^{\prime}\right)$ by a coverage factor $k=2$, to accomplish a level of confidence of $95 \%$, using the following equations:

$u^{\prime}=\sqrt{ } u^{\prime}(\text { bias })^{2}+u^{\prime}(\text { precision })^{2} ; \mathrm{U}^{\prime}=2 * \mathrm{u}^{\prime}$

\section{Statistical Analysis}

Analysis of variance (ANOVA) was carried out with the software XLSTAT (Addinsolf LTD, Version 19.4). Mean comparisons of the different solvent extractions were calculated by the Fisher's least significant difference test at $p \leq 0.05$.

\section{Results and Discussion}

The UHPLC LC-MS/MS method allowed a good separation of the selected a.is; the MRM mode showed no interfering peaks in the white wine matrix in the chromatographic range of interest even if no cleanup has been carried out on the analyzed samples, showing good selectivity of the method (Fig. 1). Among the transition selected, m/z 104 and $\mathrm{m} / \mathrm{z}$ 132 were used as quantifying ions for 2,6 TDI and 2,4 TDI, respectively, while $\mathrm{m} / \mathrm{z} 147$ was used as qualifying ion for both a.is (Table 1). The instrument limits of quantification (LOQ) calculated as 10 -fold the signal-to-noise ratio, accounted in white wine matrix for 1.72 , and $1.57 \mu \mathrm{g} / \mathrm{L}$ for 2,6 TDI and 2,4 TDI, respectively, and five points standard calibration curves from 1.72 to $1729.0 \mu \mathrm{g} / \mathrm{L}$ and from 1.57 to $1570.0 \mu \mathrm{g} / \mathrm{L}$ showed correlation coefficient $\left(R^{2}\right)$ averaging $0.9996 \pm 0.01 \%$ and $0.9992 \pm 0.08 \% 2,6$ TDI and 2,4 TDI, respectively, showing good linearity (Table 1 ).

The matrix effect, evaluated at $0.8 \mu \mathrm{g} / \mathrm{L}$, showed that the wine matrix caused a high decrease of the signal of 2,6 TDI of almost $86 \%$ while did not affected the detection of 2,4 TDI (Table 1) (Fig. 2). Therefore, quantitative analysis of the recoveries, release test, and real samples were made each with the proper calibration curve. Repeatability (RSDr) was evaluated for $n=6$, and intermediate precision (RSDWr) for $n=12$; good results were obtained for all experiments $(\mathrm{RSD} \leq 20)$ according to Eurachem CITAC (2014) with 2,6 TDI values better than that of 2,4 TDI for both spike levels. The RSDmax $\%$ was recorded in repeatability at $17.3 \%$ for 2,4 TDI RSDr at $2 \mu \mathrm{g} / \mathrm{L}$ (Table 1).

Table 1 MRM transitions and method validation parameters for 2,4 TDI and 2,6 TDI in wine matrix

\begin{tabular}{|c|c|c|c|}
\hline & & 2,6 TDI & 2,4 TDI \\
\hline \multicolumn{4}{|l|}{ MS-MRM } \\
\hline Quantitative ion, $\mathrm{m} / \mathrm{z}$ & & $175.2-104$ & $175.2-132$ \\
\hline Qualifying ion, m/z & & $175.2-147$ & $175.2-147$ \\
\hline \multicolumn{4}{|l|}{ Validation parameters } \\
\hline Regression equation & & $y=512,739.8 x+825.34$ & $y=411,920.8 x+11,335.53$ \\
\hline$R^{2} \pm \mathrm{RSD} \%$ & & $0.9996 \pm 0.01 \%$ & $0.9992 \pm 0.08 \%$ \\
\hline$U^{\prime}$ & & 13.53 & 42.05 \\
\hline r.t. (average, min.) & & 10.23 & 13.02 \\
\hline $\mathrm{LOD} \mu \mathrm{g} / \mathrm{L}$ & & 0.42 & 0.39 \\
\hline $\mathrm{LOQ} \mu \mathrm{g} / \mathrm{L}$ & & 1.72 & 1.57 \\
\hline \multirow[t]{2}{*}{$\operatorname{RSDr}(n=6) \mathrm{RSD} \%$} & $2 \mu \mathrm{g} / \mathrm{L}$ & 4.2 & 17.3 \\
\hline & $7.8 \mu \mathrm{g} / \mathrm{L}$ & 5.6 & 14.1 \\
\hline \multirow[t]{2}{*}{$\operatorname{RSDWr}(n=12) \mathrm{RSD} \%$} & $2 \mu \mathrm{g} / \mathrm{L}$ & 7.2 & 14.0 \\
\hline & $7.8 \mu \mathrm{g} / \mathrm{L}$ & 9.2 & 10.8 \\
\hline Matrix effect $\% \pm \mathrm{RSD}$ & $0.8 \mu \mathrm{g} / \mathrm{L}$ & $13.24 \pm 3.22$ & $104.05 \pm 4.91$ \\
\hline
\end{tabular}


Fig. 2 MRM chromatograms of standards of 2.6TDI and 2.4 TDI at $10 \mu \mathrm{g} / \mathrm{L}$ in (a) acid water, (b) synthetic wine, (c) ethanol, and (d) white wine

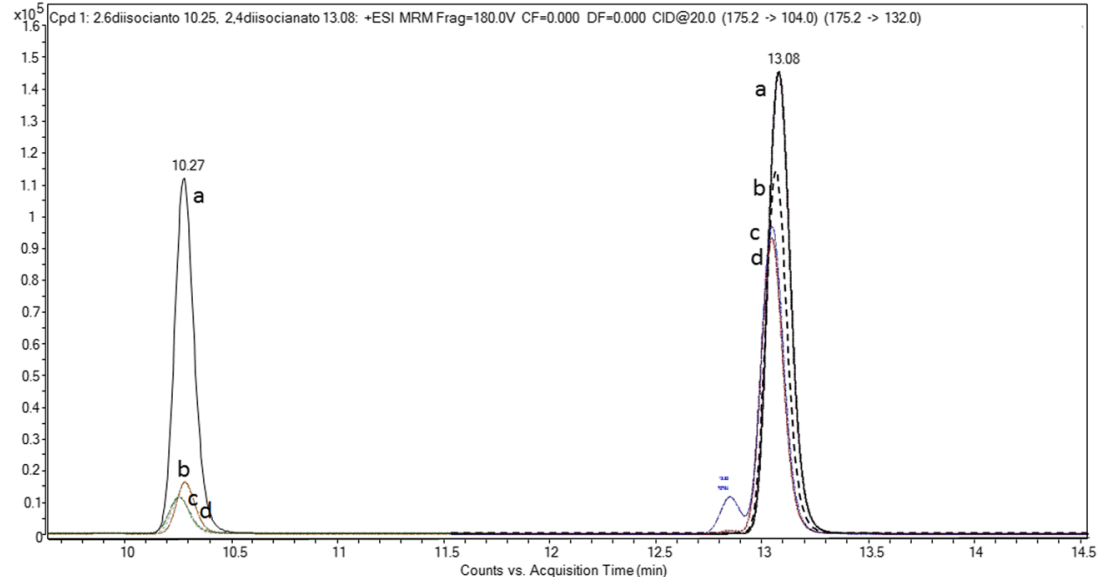

Recovery assays carried out a $10 \times$ LOQ with the four solvents showed the poor capacity of acidified water and synthetic wine to extract the a.is. from the cork powder. On the other hand, pure ethanol and wine were effective in extracting the a.is with average values of $88.53 \pm$ $3.51 \%$, and $85.11 \pm 4.01 \%$, for $2,6 \mathrm{TDI}$, and $99.18 \pm$ $3.51 \%$ and $97.90 \pm 2.46 \%$ for 2,4 TDI, respectively. Moreover, all solvents showed higher recoveries for 2,4 TDI in respect to 2,6 TDI (Table 2). Release tests performed on whole caps to simulate bottle storage conditions confirmed the higher extracting capacity of ethanol (20.15 $\pm 12.47 \%$ for 2,6-TDI and $25.61 \pm 12.46 \%$ for $2,4-$ TDI, respectively) versus white wine $(14.26 \pm 13.69 \%$ for 2,6-TDI and $16.78 \pm 14.05 \%$ for 2,4-TDI, respectively). As could be expected, the smaller specific surface area of the whole corks compared with that of the ground ones led to a lower extraction capacity and a consequent lower final residue of about four and six times less for ethanol and white wine, respectively.

The results of the analyses carried out in this work showed high differences among the thesis with lower recoveries for the 2,6 TDI especially in aqueous solutions and synthetic

Table 2 Recoveries and release test performed on cork samples spiked at $10 \times$ LOQ of the a.is

\begin{tabular}{lll}
\hline Solvents & 2,6 TDI $(\% \pm \mathrm{RSD})$ & $2,4 \mathrm{TDI}(\% \pm \mathrm{RSD})$ \\
\hline Recovery test* & $\mathrm{m} / \mathrm{z} 104$ & $\mathrm{~m} / \mathrm{z} 132$ \\
Acidified water & $17.96 \pm 15.54$ & $40.08 \pm 13.26$ \\
Synthetic wine & $22.46 \pm 6.98$ & $59.61 \pm 8.04$ \\
Ethanol 96\% & $88.53 \pm 3.51$ & $99.18 \pm 3.51$ \\
White wine & $85.11 \pm 4.01$ & $97.90 \pm 2.46$ \\
Release test & & \\
Ethanol 96\% & $20.15 \pm 12.47$ & $25.61 \pm 12.46$ \\
White wine & $14.26 \pm 13.69$ & $16.78 \pm 14.05$
\end{tabular}

* Recovery tests were made on grinded cork, release tests were made on whole cork. wine. Conversely, the recovery and release tests conducted with ethanol on both the a.is. have shown greater residues, as indeed the white wine.

In agreement with what was previously reported in literature, low residue levels obtained by acidified water and synthetic wine seem to be related to poor solubility of diisocyanate in aqueous medium leading to a minor extraction ability and their inclination to decompose with formation of primary aromatic amines (Suresh Babu et al. 2014).

Finally, six batches of real commercial micro-agglomerate cork samples have been processed using white wine and ethanol as extracting solvents to evaluate the effectiveness of the analytical method proposed in this study. The analyses showed levels of 2,6 TDI < LOD and of 2,4 TDI > LOD and $<$ LOQ in wines (Table 3). Ethanol was in all samples more effective than white wine, with values for 2,6 TDI $<$ LOQ and for 2,4 TDI ranging from $5.61 \pm 1.98 \% \mu \mathrm{g} / \mathrm{L}$ to $10.20 \pm 2.42 \% \mu \mathrm{g} / \mathrm{L}$ (Fig. 3).

Any data was found in literature concerning the release in food of TDI compounds during storage from cork stoppers.

The possible presence of toluene diisocyanate residues in the glue of micro-agglomerated cork stoppers has alarmed the American federal agencies, the Food and Drug Administration

Table 32,4 TDI and 2,6 TDI residues ( $\mu \mathrm{g} / \mathrm{L} \pm \mathrm{RSD} \%$ ) in commercial samples of micro-agglomerate cork extracted with ethanol and white wine

\begin{tabular}{llllll}
\hline Samples & \multicolumn{2}{l}{$2,6 \mathrm{TDI}$} & & & \multicolumn{2}{l}{$2,4 \mathrm{TDI} \mu \mathrm{g} / \mathrm{L} \pm \mathrm{RSD} \%$} \\
\cline { 2 - 3 } \cline { 6 - 7 } & Wine & Ethanol & & Wine & Ethanol \\
\hline $\mathrm{C} 1$ & $<$ LOD & $<$ LOD & & $<$ LOQ & $6.12 \pm 2.01$ \\
$\mathrm{C} 2$ & $<$ LOD & $<$ LOD & & $<$ LOQ & $10.2 \pm 3.13$ \\
$\mathrm{C} 3$ & $<$ LOD & $<$ LOD & & $<$ LOQ & $8.24 \pm 2.42$ \\
$\mathrm{C} 4$ & $<$ LOD & $<$ LOD & & $<$ LOD & $<$ LOQ \\
C5 & $<$ LOD & $<$ LOD & & $<$ LOQ & $5.61 \pm 1.98$ \\
C6 & $<$ LOD & $<$ LOD & $<$ LOD & $<$ LOD \\
\hline
\end{tabular}


Fig. 3 MRM chromatograms of 2.6TDI and 2.4 TDI in real samples extracted with ethanol, (a) sample C5, (b) sample $\mathrm{C} 2$

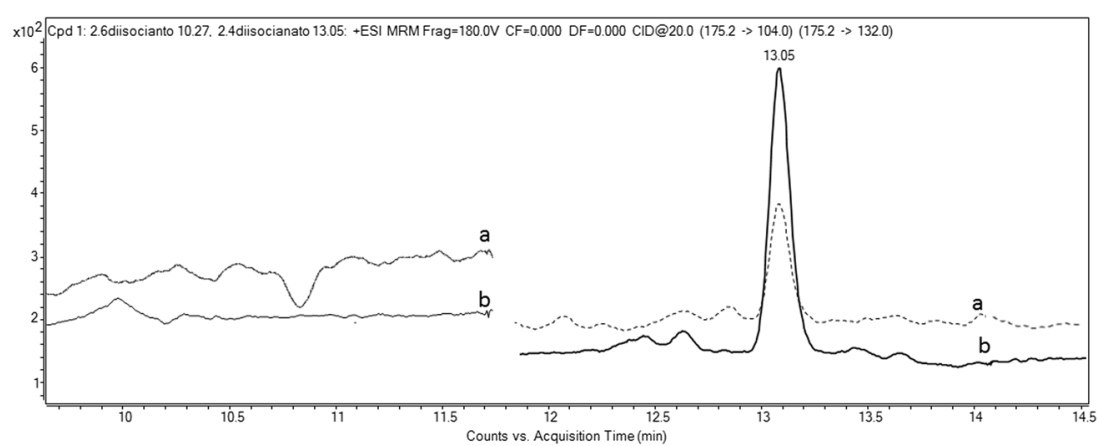

(FDA) and the Environmental Protection Agency (EPA), as these substances are suspected to be potentially carcinogenic. Despite the producers say that the residues are extremely low and there are no toxicological problems in the USA, this problem could be quite serious given the high consumption of these caps which is around 350 million bottles per year.

Given the established dermal- and inhalation-sensitizing capacity of TDI, in January 2015, the EPA proposed rules of use and caution, the Significant New Use Rule (SNUR) under the control of the Toxic Substances Control Act (TSCA) for the use of seven TDIs.

These rules give the authority to the EPA to evaluate and in the event of making decisions to prohibit or limit the use of these chemicals at concentrations greater than $0.1 \%$ on adhesives, coatings, elastomers, binders, and sealants in consumer products, including imported consumer products.

The action plan concerns the use of toluene diisocyanate (TDI) and related compounds in products to which the consumer and more generally the population could be exposed, in or around sensitive buildings such as houses and schools.

The toxicological data available on TDI exposure are related to primary users and bystanders; moreover, with a shortterm exposure of $0.02 \mathrm{mg} / \mathrm{kg}$, a particular attention is devoted to children when the exposition comes from polluted air. Total concentration of monomeric isocyanates in plastic materials which can be in contact with foodstuffs must be below 1 $\mathrm{mg} / \mathrm{kg}$ food-contact material (Directive 2002/17/EC 2002).

Six and Feigenbaum (2003) has reported a comprehensive study of possible migrants from synthetic products used for cork production, pointing out the need to carry out residue check control, even if regulatory requirements are not available.

The data from real samples showed a zero or very low migration of TDI compounds in the wine; therefore, a risk assessment allowed to say that from a risk management point of view, there is no toxicological risk.

\section{Conclusion}

In this paper, it has been developed and validated a simple and rapid method for the determination of 2,6 TDI and 2,4 TDI in micro-agglutinated cork samples in wine. Validation parameters were in agreement with the Eurachem CITAC (Magnusson and Örnemark 2014) values for contaminants in food matrices. The LOQ of the method allowed to quantify residues at concentrations of 1.72 and $1.57 \mu \mathrm{g} / \mathrm{L}$, for 2,6 TDI and 2,4 TDI, respectively (Table 1).

This study showed the high individual variability of real samples from different batches, and moreover, the analysis showed that the migration from the micro-agglomerate corks in bottle during shelf-life is very low and do not create a toxicological alarm.

Authors' Contributions Francesco Corrias has carried out formal analysis by UHPL-MS/MS, and manuscript drafting and reviewing.

Eleonora Cossu has carried out sample preparation, recovery, release test, and real sample analysis

Paolo Cardu has participated in the conceptualization of the research and the supervision of sample processing.

Alberto Angioni has participated in the conceptualization and methodology development; moreover, he made the validation protocol and data analysis, wrote the original paper, reviewed, and edited.

Funding Open access funding provided by Università degli Studi di Cagliari within the CRUI-CARE Agreement.

\section{Compliance with Ethical Standards}

Conflict of Interest Francesco Corrias declares that he has no conflict of interest. Eleonora Cossu declares that she has no conflict of interest. Paolo Cardu declares that he has no conflict of interest. Alberto Angioni declares that he has no conflict of interest.

Informed Consent Not applicable.

Open Access This article is licensed under a Creative Commons Attribution 4.0 International License, which permits use, sharing, adaptation, distribution and reproduction in any medium or format, as long as you give appropriate credit to the original author(s) and the source, provide a link to the Creative Commons licence, and indicate if changes were made. The images or other third party material in this article are included in the article's Creative Commons licence, unless indicated otherwise in a credit line to the material. If material is not included in the article's Creative Commons licence and your intended use is not permitted by statutory regulation or exceeds the permitted use, you will need to obtain permission directly from the copyright holder. To view a copy of this licence, visit http://creativecommons.org/licenses/by/4.0/. 


\section{References}

Azevedo J, Fernandes I, Lopes P, Roseira I, Cabral M, Mateus N, Freitas V (2014) Migration of phenolic compounds from different cork stoppers to wine model solutions: antioxidant and biological relevance. Eur Food Res Technol 239:951-960

CD-P-MCA (2007) European Committee for Food Contact Materials and Articles. Policy statement concerning. Cork stoppers and other cork materials and articles intended to come into contact with foodstuff. Version 2, 05/09/2007

COMMISSION DIRECTIVE 2002/17/EC of 21 February 2002, amending Directive 90/128/EEC relating to plastic materials and articles intended to come into contact with foodstuffs

Courtney S (2001) The history and revival of screwcaps. Wine of the week. https://www.wineoftheweek.com/screwcaps/history.html. Accessed 19 April 2020

Denecker E, Vandorpe K (2007) Sealed amphora stoppers and tradesmen in Greco-Roman Egypt: archaeological, papyrological and inscriptional evidence. BABesch 82:115-128. https://doi.org/10.2143/ BAB.82.1.2020764

Donchenko A, Aubin S, Gagné S, Spence M, Breau L, Lesage J (2020) Development of a method for quantification of toluene diisocyanate and methylenediphenyl diisocyanate migration from polyurethane foam sample surface to artificial sweat by HPLC-UV-MS. J Chromatogr B 1142:122027

EPA, U.S (2011) Environmental Protection Agency, Toluene Diisocyanate (TDI) and related compounds Action Plan [RIN 2070/ZA14]

FDA (2017) Food and Drug Administration, Environmental Memorandum - FCN 1854 FONSI, Mariellen Pfei

Gagné S, Lesage J, Ostiguy C, Van Trab H (2003) Determination of unreacted 2,4-toluene diisocyanate (2,4TDI) and 2,6-toluene diisocyanate $(2,6 \mathrm{TDI})$ in foams at ultratrace level by using HPLCCIS-MS-MS. Analyst. 128:1447-1145

Gil L (2014) Cork: a strategic material. Front Chem.2:16. Published online 2014 Apr 11. https://doi.org/10.3389/fchem.2014.00016

Gil L, Pereira C, Branco P, Teixeira A (2006) Formation of acutissimin A in red wine through the contact with cork. J Int Sci Vigne Vin 40: 217-221

Godden P, Francis IL, Field J, Gishen M, Coulter A, Valente P, Hoj P, Robinson E (2008) Wine bottle closures: physical characteristics and effect on composition and sensory properties of a Semillon wine I. Performance up to 20 months post-bottling. Aust J Grape Wine Res 7(2):64-105. https://doi.org/10.1111/j.1755-0238.2001. tb00196.x

He J, Zhou Q, Peck J, Soles R, Qian MC (2013) The effect of wine closure on volatile sulfur and other compounds during post-bottle ageing. Flavour Fragr J 28(28):118-128

IARC (1986) IARC monographs on the evaluation of the carcinogenic risk of chemicals to humans: some chemicals used in plastics and elastomers. IARC Monograph 39. Lyon, France: World Health
Organization, International Agency for Research on Cancer, pp. 287-323

ISO 633:2019. https://www.iso.org/obp/ui/\#iso:std:iso:633:ed-3:v1:en. Accessed 19 April 2020

Magnusson B, Örnemark U (eds.) (2014) Eurachem CITAC Guide: the fitness for purpose of analytical methods - a laboratory guide to method validation and related topics, (2nd ed. 2014). ISBN 97891-87461-59-0. Available from https://www.eurachem.org. Accessed 19 April 2020

Nasini A, Smedile S (2012) Technical manual for the use of cork stoppers. 2012. Noesis Comunicazione, Milan

Pereira AC, Brezet H, Pereira H, Vogtlander J (2012) Cork and sustainability: discussing the sustainable use of the material from a design perspective. J Shanghai Jiaotong Univ (Sci) 17(3):360-363. https:// doi.org/10.1007/s12204-012-1287

Prak S, Gunata Z, Guiraud JP, Schorr-Galindo S (2007) Fungal strains isolated from cork stoppers and the formation of 2,4,6trichloroanisole involved in the cork taint of wine. Food Microbiol 24(3):271-280

SANTE/E4/VW 10235/2016 - Rev. 4, Commission working document, on the evaluation of data submitted to confirm MRLs. following the review of existing MRLs. Brussels, 18 February 2020

Six T, Feigenbaum A (2003) Mechanism of migration from agglomerated cork stoppers. Part 2: safety assessment criteria of agglomerated cork stoppers for champagne wine cork producers, for users and for control laboratories. Food Addit Contam 20(10):960-971

Six T, Feigenbaum A, Riquet AM (2002) Mechanism of migration from agglomerated cork stoppers: I. An electron spin resonance investigation. J Appl Polym Sci 83:2644-2654. https://doi.org/10.1002/ app. 10230

Suresh Babu KV, Rama Teja AS, Srinivas Babu N, Arunachalam V, Maheswar CVS, Audisesha Reddy K (2014) Methods of decontamination of toluene di-isocyanate (TDI) spills and leftovers. Res J Chem Sci. 4(4):89-93. Available online at www.isca.in. Accessed 19 April 2020

Wirth J, Caillé S, Souquet JM, Samson A, Dieval JB, Vidal S, Fulcrand H, Cheynier V (2012) Impact of post-bottling oxygen exposure on the sensory characteristics and phenolic composition of Grenache rosé wines. Food Chem 132(4):1861-1871, 6th International Conference on Water in Food. https://doi.org/10.1016/j.foodchem. 2011.12.019

Yeh HJ, Shih TS, Tsai PJ, Chang HY (2002) Toluene diisocyanate concentration investigation among TDI-related factories in Taiwan and their relations to the type of industry. J Expo Anal Environ Epidemiol 12:157-164

Yun JS, Park JH, Lee KM, Choi HS, Cho YB, Koh SB, Cha BS (2006) Gas Chromatographic analysis of TDI, MDI and HDI using 2chlorobenzyl alcohol and 2,4-dichlorobenzyl alcohol derivatives. J Korean Soc Occup Environ Hygn 16(3):222-232

Publisher's Note Springer Nature remains neutral with regard to jurisdictional claims in published maps and institutional affiliations. 